\title{
Parafoil Control Authority with Upper-Surface Canopy Spoilers
}

\author{
Alek Gavrilovski,* Michael Ward,^ and Mark Costello \\ Georgia Institute of Technology, Atlanta, Georgia 30332 \\ DOI: $10.2514 / 1 . C 031685$
}

\begin{abstract}
Current autonomous parafoil and payload aircraft are controlled by deflection of the canopy trailing edge. Asymmetric trailing edge deflection results in a turn, symmetric deflection results in a change in speed with little change in glide slope. The inability to control glide slope with these traditional canopy control mechanisms is severely detrimental to the landing accuracy of parafoil and payload aircraft, especially when turbulent atmospheric conditions or difficult terrain are present near the landing area. An alternative canopy control mechanism is investigated where internal air is vented through the upper surface of the canopy to create an aerodynamic spoiler. Flight-test results for various configurations of the spoiler concept are presented. It is shown that a dramatic change in glide slope can be generated with relatively small spoiler actuations. Furthermore, it is shown that the same lateral control authority available with trailing edge brakes can be achieved with asymmetric actuation of the upper-surface spoilers.
\end{abstract}

\section{Introduction}

A IRDROP systems offer the unique capability of delivering large payloads to undeveloped and inaccessible locations. Traditionally, these systems have been unguided and, consequently, either a large landing zone is required or a high probability of losing individual payloads must be accepted. Autonomous-guided airdrop systems based on steerable, ram-air parafoils were developed with the goal of improving the precision and accuracy of air-dropped payload delivery. These systems use trailing edge (TE) brake deflection for control [1-9]. Differential brake deflection produces lateral control. Symmetric brake deflection predominantly causes a reduction in flight speed with small changes in the glide slope until stall. Typical flight control laws for guided parafoil systems use only lateral control and achieve a limited degree of glide slope control using an altitude-dump maneuver in the form of a series of S-turns. This method of terminal guidance is susceptible to atmospheric gusts and surface conditions at the target area and can induce significant errors in the final landing position. Numerous researchers have developed parafoil control schemes using right and left brakes as the control mechanism. The algorithms have become steadily more sophisticated and have achieved better accuracy. However, with the current TE deflection control paradigm, it appears that a point of diminishing return is being reached in terms of landing accuracy.

The addition of glide slope control has been shown to be a powerful means to increase landing accuracy. Slegers et al. [10] demonstrated effective glide slope control by dynamically varying the canopy incidence angle and estimated a factor of three improvements in landing accuracy in simulation. This was accomplished by varying the length of the leading edge (LE) risers in concert with the TE brakes, thereby, rotating the canopy longitudinally and controlling the trim angle of attack directly in flight. Another means for achieving glide slope control common to fixed-wing aircraft is the activation of spoilers on the upper surface of a wing. Sailplanes have traditionally used this control mechanism for altering speed and glide slope on landing approach [11]. In the 1960s, spoiler devices were incorporated

Presented at the AIAA Aerodynamic Decelerator Conference, Dublin, Ireland, May 24-26, 2011; received 27 September 2011; revision received 30 January 2012; accepted for publication 31 January 2012. Copyright ( 2012 by Mark Costello. Published by the American Institute of Aeronautics and Astronautics, Inc., with permission. Copies of this paper may be made for personal or internal use, on condition that the copier pay the $\$ 10.00$ per-copy fee to the Copyright Clearance Center, Inc., 222 Rosewood Drive, Danvers, MA 01923; include the code 0021-8669/12 and $\$ 10.00$ in correspondence with the CCC.

${ }^{*}$ Graduate Research Assistant, School of Aerospace Engineering, 270 Ferst Drive, Atlanta, GA 30332. Student Member AIAA.

${ }^{\dagger}$ Sikorsky Associate Professor, School of Aerospace Engineering, 270 Ferst Drive, Atlanta, GA 30332. Associate Fellow AIAA. on powered aircraft for direct lift control. Kohlman and Brainerd [12] demonstrated the benefits of using upper-surface aerodynamic spoilers for glide path control on light aircraft.

The research reported in this paper examines an entirely new mechanism for control of ram-air parafoil canopies based on the idea of using upper-surface spoilers as both glide slope and turn rate control mechanisms. Rather than attempt to incorporate a mechanical flap into the canopy, ram air is vented through slits in the upper surface to create an effective aerodynamic spoiler. The ability of the upper-surface-slit spoiler to generate glide slope change and provide turn rate control is examined via a flight-test program using a small powered parafoil and payload system. A parametric study of the construction geometry, size, and location of the upper-surface spoilers is given to provide a set of design guidelines for the incorporation of this new control mechanism in parafoil systems. The paper begins with a detailed description of the control mechanism, which is followed by a description of flight hardware and flight operation. Next, the data processing of flight measurements is discussed. Finally, flight-test results for glide slope control and turn rate control are presented for a variety of spoiler configurations.

\section{Upper-Surface Canopy Spoiler Concept}

A schematic of the upper-surface spoiler concept is shown in Fig. 1. On the top surface of the canopy, a spanwise slit is introduced across a number of cells in the center section of the wing. The slit location was determined using computational fluid dynamics simulations as a guide and corresponds to the minimum pressure point on the upper surface. All the cells that contain a slit have a control line attached to the LE side of the slit. These lines pass through the bottom surface before joining and connecting to a single winch servo. When the winch servo actuates the control line, the material ahead of the slit is deflected downward. The remainder of the cell on the TE side of the slit remains unperturbed, due to the internal pressure of the canopy. This causes an airflow bubble on the upper surface, which distorts the airflow, much like conventional aircraft spoilers except that this slit spoiler configuration uses vented ram air as a spoiler rather than a mechanical flap. When the slit is not actuated, the spanwise tension in the canopy is sufficient to keep the slit closed. Figure 2 shows the slit control mechanism as implemented on the $2.7 \%$ canopy used for flight testing. The slit connections are attached to a single winch servo using a cascaded control line. The actuation of the spoiler produces the openings, and the final spoiler shape is shown in the second part of Fig. 2 .

\section{Flight-Test Description}

The control authority of the upper-surface-slit spoiler concept was evaluated experimentally. The data for the results presented below 


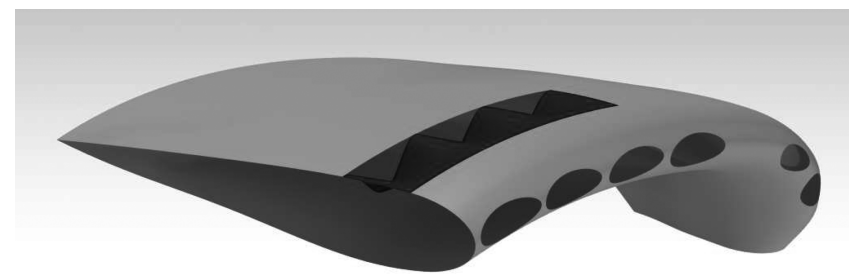

Fig. 1 Upper-surface-slit spoiler concept.
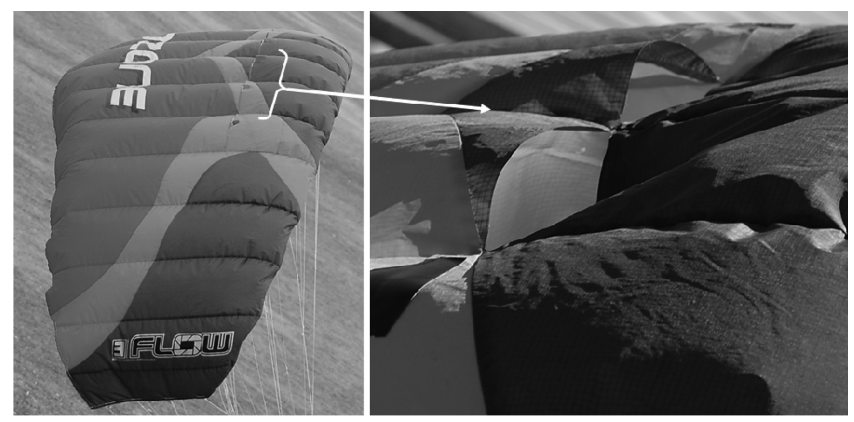

a) Unactuated slit spoiler

b) Actuated slit spoiler detail

Fig. 2 Upper-surface-slit spoiler on test flight canopy.

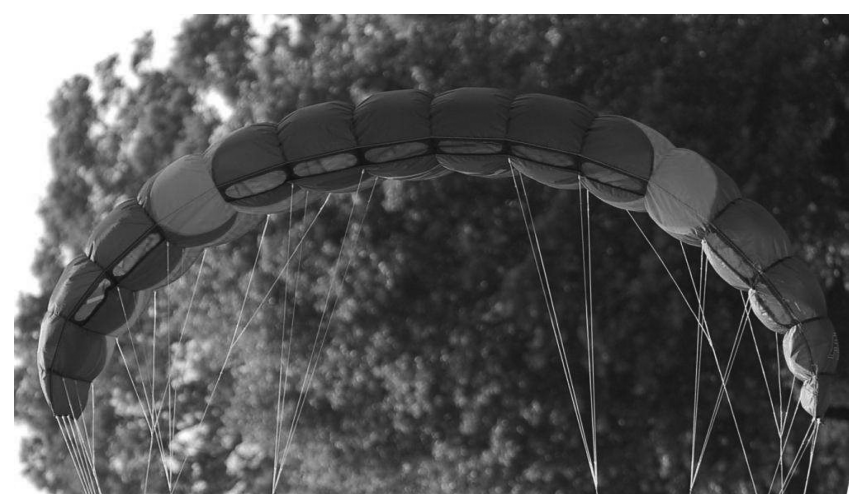

Fig. 3 Front view of tested canopy.

was gathered during approximately 20 flights. To obtain high-quality data, test flying was conducted only in very calm, atmospheric conditions (normally just after sunrise, right before sunset, or at night).
The canopy used for testing is shown in Fig. 3 . It is a $2.7 \mathrm{~m}^{2}$ paragliding-style canopy with 16 cells. The center eight cells compose $56 \%$ of the constructed span and $68 \%$ of the projected span. The canopy has a constructed aspect ratio of 3.35 and a projected aspect ratio of 2.4. The canopy has an elliptical planform with a root chord of $1.1 \mathrm{~m}$ and a tip chord of $0.4 \mathrm{~m}$. The constructed wingspan is $3.12 \mathrm{~m}$ and the projected span is $2.52 \mathrm{~m}$.

The canopy was flown with a self-powered payload shown in Fig. 4. The payload mass is $3.8 \mathrm{~kg}$, resulting in a wing loading of $1.4 \mathrm{~kg} / \mathrm{m}^{2}\left(0.29 \mathrm{lb} / \mathrm{ft}^{2}\right)$. The system has a nominal glide ratio of 3.8 and a nominal airspeed of $8 \mathrm{~m} / \mathrm{s}$.

The payload consists of a wooden frame to which the flight components are attached. The wheeled landing gear and a 10-cc $\left(0.60 \mathrm{in}^{3}\right)$ model airplane engine allow for self-powered flight with rolling takeoffs. Three main servos are used to achieve basic control of the system. Two servos attach to different halves of the TE and are used for steering and braking in the conventional manner. The LE lines are attached to a single servo and are used for incidence angle control in conjunction with symmetric brake deflection. Spoiler actuation is performed with an auxiliary winch servo. The instrument package includes a Global Positioning System (GPS) sensor, barometric altimeter, accelerometers, rate gyros, and magnetometer. During data acquisition, the payload is controlled through the instrument package. Both payload position and control actuation are recorded in the output data. A set of lights provide illumination for night flying. The fuel tank can hold a maximum of half a pound of fuel, which is used up completely during the climb to the test altitude. This ensures consistent payload weight between tests. Using this scheme data acquisition runs from up to $4000 \mathrm{ft}$. Above ground level can be performed.

A typical flight-test profile is illustrated in Fig. 5. Takeoff is followed by a powered climb to high altitude, during which the payload is configured for optimal climb performance (dashed line). The climb is terminated when the fuel supply is exhausted. Data acquisition is initiated while the system is in gliding flight (dotted line in figure). When sufficient data is collected, or the payload descends to a low altitude, the data acquisition is terminated and a manual landing is performed. This flight-test setup eliminates the issues of canopy deployment from free-fall, reduces overall cost, and reduces the time between test runs.

For the purpose of glide slope estimation, the ratio of forward velocity to vertical velocity is needed. The computation of the forward velocity of the vehicle relative to the air mass requires the knowledge of the atmospheric wind conditions. To achieve this, test flights are conducted with the vehicle flying at a constant and small turn rate. Figure 6 shows typical position data obtained during a single flight using GPS. This particular flight includes three data

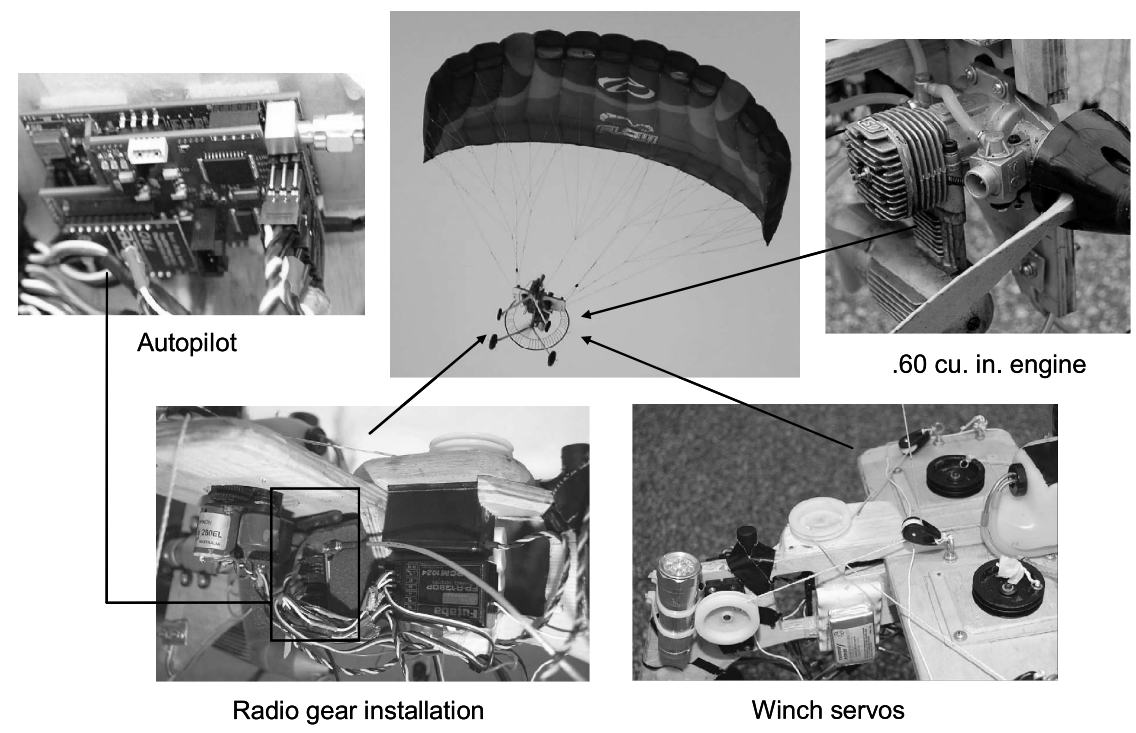

Fig. 4 Flight-test hardware. 


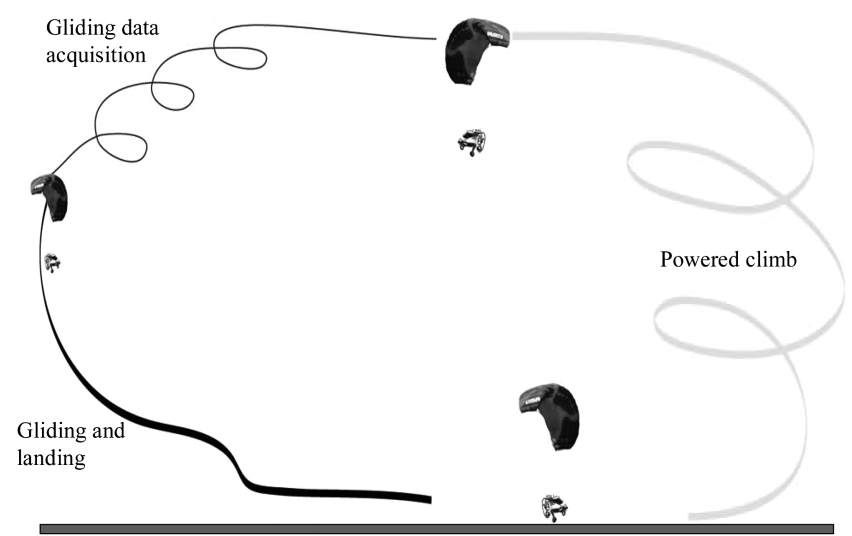

Fig. 5 Flight-test profile.

acquisition sequences. The drift in the ground tracks for each sequence in Fig. 6 indicate the wind vector. The total vehicle velocity then contains sinusoidal variations due to the wind, allowing simple estimation of the horizontal airspeed, shown in Fig. 7.

The vertical component of airspeed is obtained by differentiating altitude data from a barometric altimeter. Barometric altimeter data is useful since it is available at a higher frequency than the GPS measurement and is more precise than GPS in the vertical direction. This yields sufficient information to estimate the glide slope of the vehicle. In addition, the control inputs are recorded and a relationship between glide slope and control input can be established.

The turn rate is estimated by differentiating the heading angle information extracted from the GPS data. This turn rate data is used to

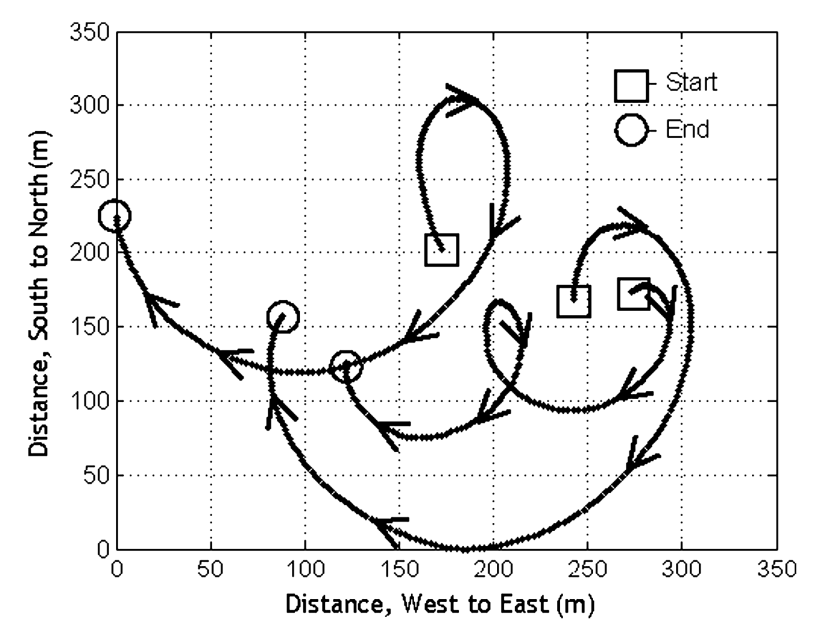

Fig. 6 Example ground tracks generated during one flight.

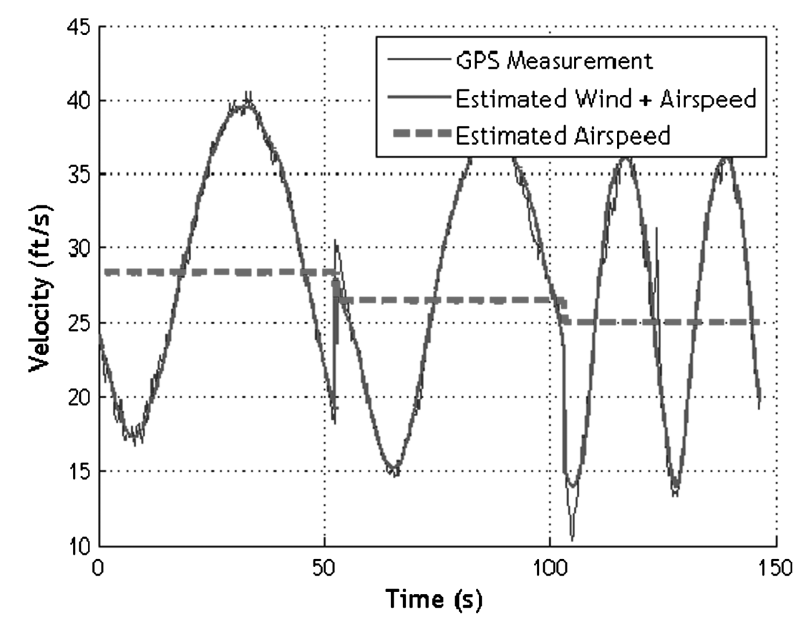

Fig. 7 Extracting horizontal airspeed from velocity data. evaluate the lateral control authority of the asymmetric spoiler configurations. A simple average of the turn rate obtained from a long segment of constant control input is used to determine the steadystate turn rate resulting from a particular spoiler configuration and deflection.

The airspeed, glide slope, and turn rate estimates are all determined by taking the average over a set of measurements, so confidence intervals may be easily calculated to yield an uncertainty estimate for each data point. For a data segment of length $n$, with mean $x_{\text {mean }}$ and sample standard deviation $s$, a conservative estimate of the $95 \%$ confidence interval for the estimated quantity $x$ is obtained according to Eq. (1) [13]:

$$
x=x_{\text {mean }} \pm 2 s / \sqrt{n}
$$

Due to the extremely time-consuming nature of the flight-testing process, only nine of the canopy configuration and control input combinations tested were repeated to verify these confidence interval estimates. While repeated estimates of turn rate fall within the estimated uncertainty levels, the variation in repeated airspeed and glide slope estimates is significantly higher than what the confidence intervals predict. This is thought to be primarily a result of the sensitivity of the airspeed and glide slope estimates to atmospheric winds. In particular, the atmospheric wind does not vary instantaneously in time, implying that disturbances to the airspeed are correlated in time. This means that the assumption of independent disturbances for each measurement assumed during the confidence interval calculation is not valid, resulting in an underestimate of the actual uncertainty level. To provide a better estimate of the uncertainty levels for airspeed and glide slope, confidence intervals are estimated directly from the observed variation in the repeated data points. In a similar method to the standard confidence interval calculation, the uncertainty bounds are calculated as twice the standard deviation of the difference in the repeated airspeed and glide slope estimates. The resulting error bounds are $\pm 8 \%$ for repeated airspeed estimates and $\pm 11 \%$ for repeated glide slope estimates. These error bounds are used as the confidence intervals for all of the presented airspeed and glide slope estimates. Confidence intervals for the turn rate estimates are calculated for each data point according to Eq. (1).

\section{Results}

Parametric studies of both symmetric and asymmetric spoiler configurations were conducted experimentally. The goal of the symmetric spoiler is to reduce glide slope in a controllable manner, and the goal of the asymmetric spoiler is to produce a turn rate. A limit on control travel is imposed by the local cell height at the spoiler location. When the upper surface is maximally actuated, the upper and lower surfaces of the canopy come in contact. It was observed in the flight test that actuation up to approximately $90 \%$ of this limit began to cause large canopy deformations (of the lower surface, as well as the upper surface) and possibly stall, so the actual limit on spoiler deflection was configuration dependent and is discussed below. All spoiler deflections are presented as fractions of the cell height at the chordwise location of the spoiler slit. The cell heights at the 15,30 , and $50 \%$ chord locations are $17.2,17.7$, and $13.5 \mathrm{~cm}$, respectively.

\section{A. Symmetric Spoiler Configurations}

A parametric study of symmetric spoiler configurations was performed by varying the construction, span, and chordwise position of the upper-surface slits. The construction of the slits was varied by moving the attachment points from the LE side of the slits to the TE side and by testing the effect of adding sealing flaps to the slits. All of these configurations have slits across the center eight cells at the $30 \%$ chord location. The span of the spoilers was varied by testing configurations with slits across the center two, four, six, and eight cells, resulting in spoiler widths of $14,28,42$, and $56 \%$ of the constructed span. All of these slits were made at the $30 \%$ chord line. 
The effect of chordwise location was tested using configurations with slits across the center eight cells at 15,30 , and $50 \%$ chord locations.

The first basic construction variation tested was the location of the spoiler actuation line attachment. The actuation of the LE side of the slit causes the fabric in front of the slit to deflect downward while leaving the fabric aft of the slit in its original shape, as shown in Fig. 2. This causes the air to be vented from the canopy in an opposing direction to the freestream airflow. The actuation of the TE side of the slit causes the fabric aft of the slit to be deformed while leaving the fabric in front of the slit in its original shape, venting air along the direction of the freestream flow. This situation is depicted in Fig. $\underline{8}$.

The second construction variation was the addition of internal flaps to help seal the slits when the spoilers are not actuated. The sealing flaps effectively extend the fabric on the LE side of the slit as shown in Fig. 9. When the spoilers are not actuated, the internal pressure presses the flap against the slit and creates a seal, preventing any air from venting into the freestream and disturbing the airflow over the upper surface. When actuated, the shape of the opening is very similar to that of the simple slit, except that slightly more actuation is required to make up for the extra length of the flap.

Flight-test results comparing these variations in spoiler construction are shown in Fig. 10. Overall, the deflection of the spoiler produces a reduction in lift, resulting in a lower value for glide slope. Glide slope reduction occurs in a smooth fashion up to the maximum deflection. The descent rate is increased, and the forward velocity is decreased, resulting in a net reduction in airspeed.

Comparing the results for LE vs. TE actuation, it is clear that actuation of the LE of the slit is more effective in reducing glide slope. The actuation of the LE allows a $64 \%$ reduction in glide slope compared to a $43 \%$ reduction when actuated the TE of the slit. Also, the canopy maintains a significantly higher airspeed under the actuation of the TE until the actuation reaches $90 \%$ of the cell height,

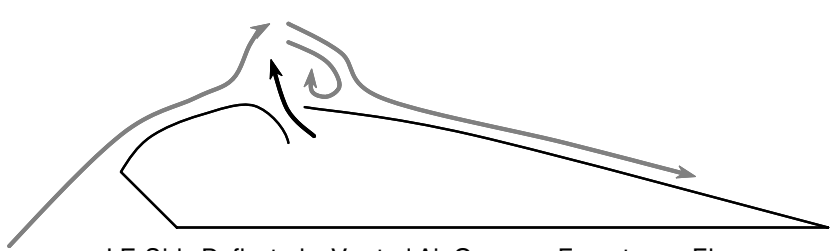

LE Side Deflected - Vented Air Opposes Freestream Flow

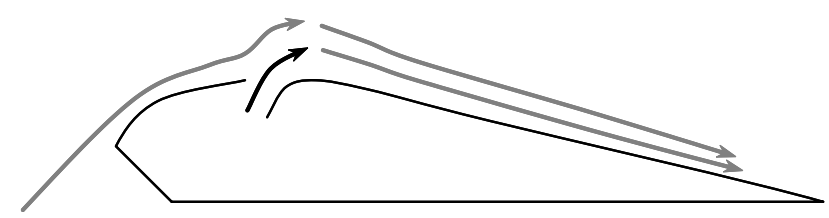

TE Side Deflected - Vented Air Directed Along Freestream Flow

Fig. 8 LE vs. TE deflection of slit.
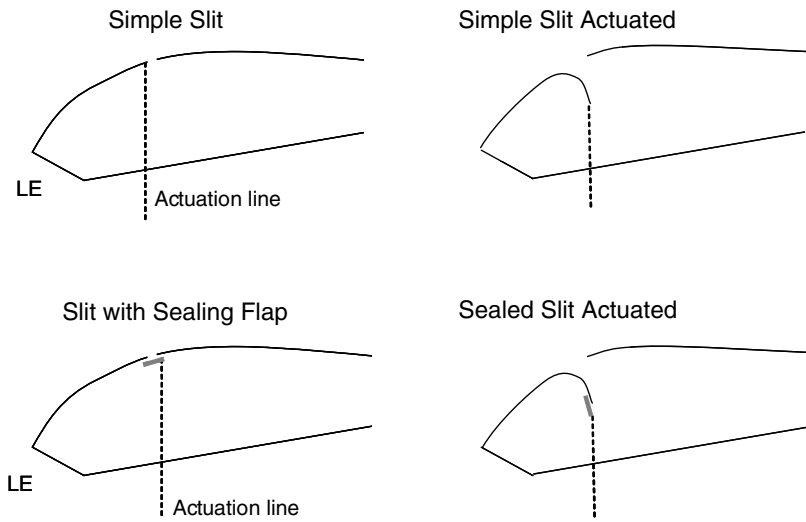

Sealed Slit Actuated

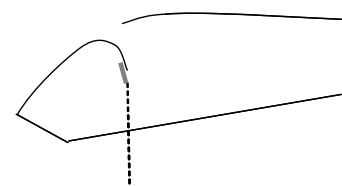

Fig. 9 Addition of sealing flaps to simple slits.
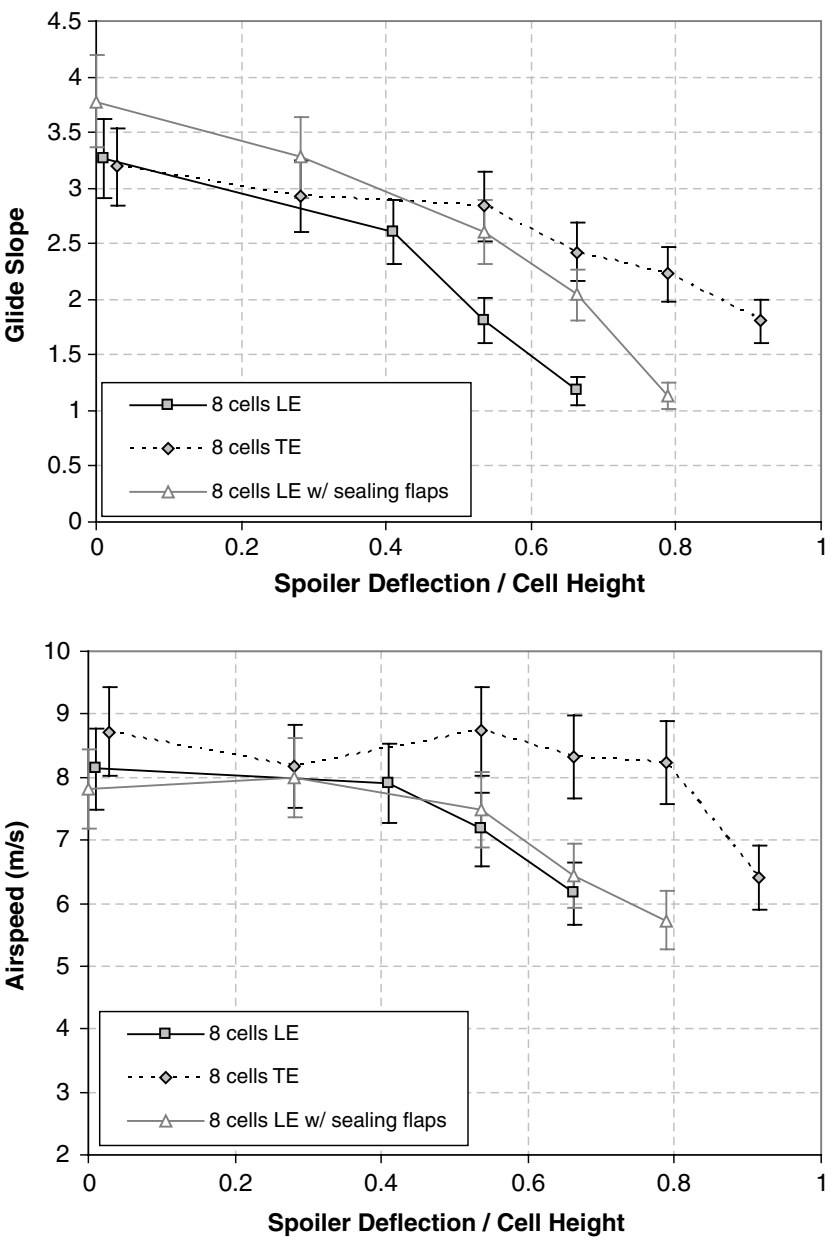

Fig. 10 Effects of varying spoiler construction.

where there is a sudden drop in airspeed. This is most likely due to large-scale canopy deformation caused by the extreme actuation of the spoiler.

Comparing the simple slit and sealed slit construction with the actuation of the LE side, it is apparent that the nominal glide slope corresponding to zero spoiler actuation is slightly reduced due to air leaking through the simple slits. When sealing flaps are incorporated into each slit, the nominal glide slope loss is recovered and the zero spoiler deflection glide slope increases from 3.2 to 3.8. As expected, the minimum glide slope achieved with the sealed slit design is the same as that achieved with the simple slits, though a slightly higher spoiler deflection is required to take up the extra fabric introduced by the sealing flaps. By improving the seal of the closed slits, the sealing flaps increase the effective control authority of the spoilers from a $64 \%$ reduction in glide slope to a $70 \%$ reduction.

The effect of spoiler span on the glide slope and airspeed control authority is shown in Fig. 11. All of these configurations use simple, unsealed slits with LE actuation. As expected, the effectiveness of the spoiler increases as the span is increased. With two cells, the nominal glide slope of 3.2 can be reduced to 2.5 , and with eight cells, the glide slope can be reduced to 1.1. The general trend in airspeed is that there is a reduction in airspeed proportional to the reduction in glide slope. With eight cells actuated to produce a $64 \%$ reduction in glide slope, there is a corresponding reduction in airspeed of $25 \%$. For comparison, with the heavy deflection of the TE brakes, the same canopy experiences a $30 \%$ reduction in airspeed with only a $0.5 \%$ reduction in glide slope.

Figure 12 depicts the growth in spoiler effectiveness by increasing the number of cells containing actuated slits. The maximum spoiler deflection for the two- to six-cell configurations was determined by the observation of significant canopy deformation. For the eight-cell configuration, the maximum spoiler deflection was determined by the flight condition. At lower glide ratios, the system became difficult 

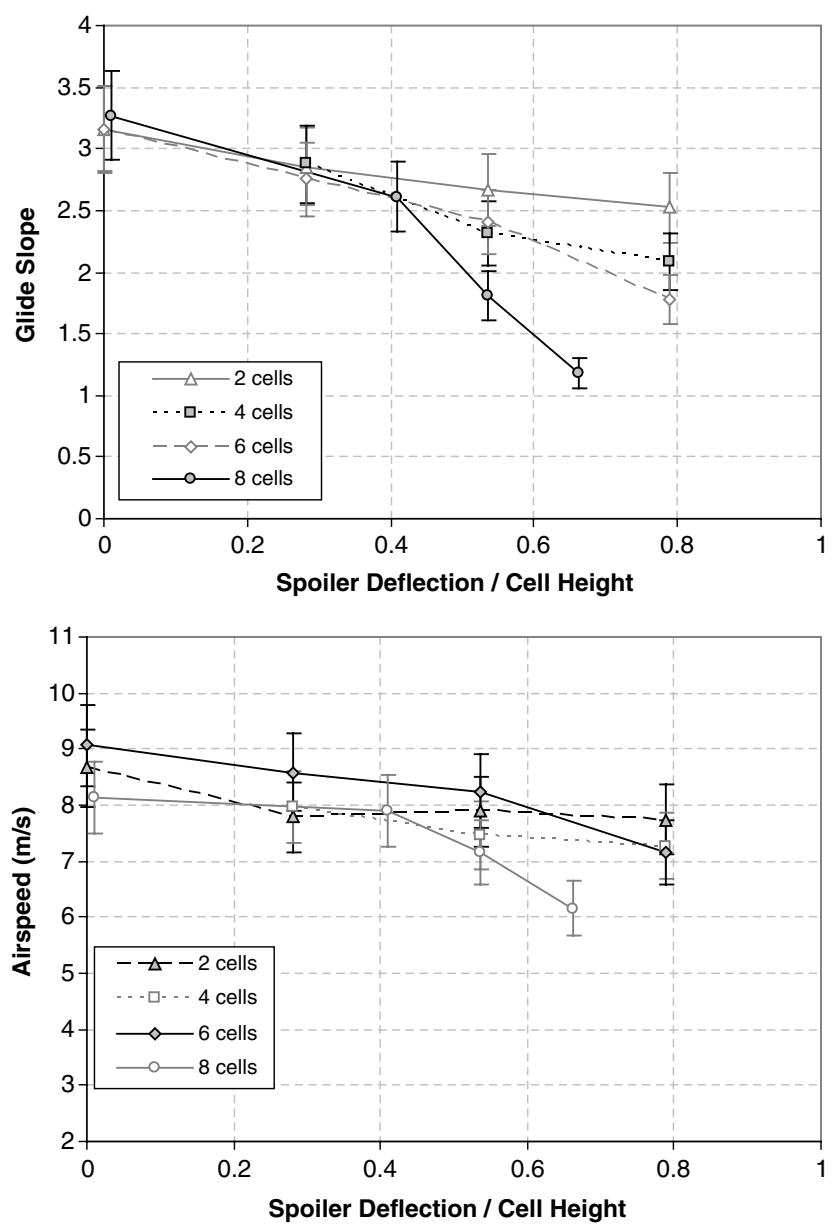

Fig. 11 Effect of varying spoiler span.

to control and prone to stall, implying that a minimum practical glide ratio limit for this particular canopy was reached. This means that increasing the span of the spoiler beyond eight cells will not increase the range of glide slope control authority. In addition, the canopy becomes increasingly sensitive to small variations in the slit and actuation line construction as the spoiler span is increased, which suggests that, practically speaking, the spoiler span should not be increased beyond the minimum span necessary to reach the minimum glide ratio limit of a particular canopy's flight envelope.

The final variation in symmetric spoiler configuration examined was the chordwise location of the upper-surface slits. The effect of moving the slit locations aft from 30 to $50 \%$ of the canopy chord on glide slope and airspeed is shown in Fig. 13. Both configurations had simple, unsealed slits across eight cells. The slits at $50 \%$ chord are less effective than the slits at $30 \%$ chord. A similar reduction in glide slope and corresponding reduction in airspeed are obtained with the

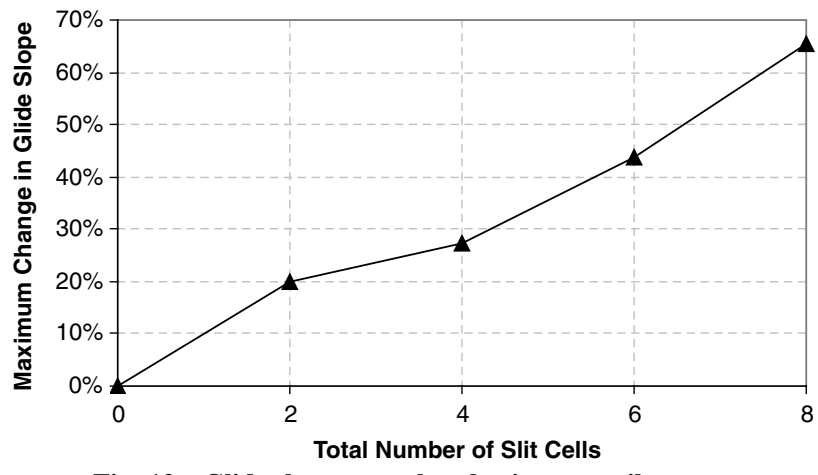

Fig. 12 Glide slope control authority vs. spoiler span.
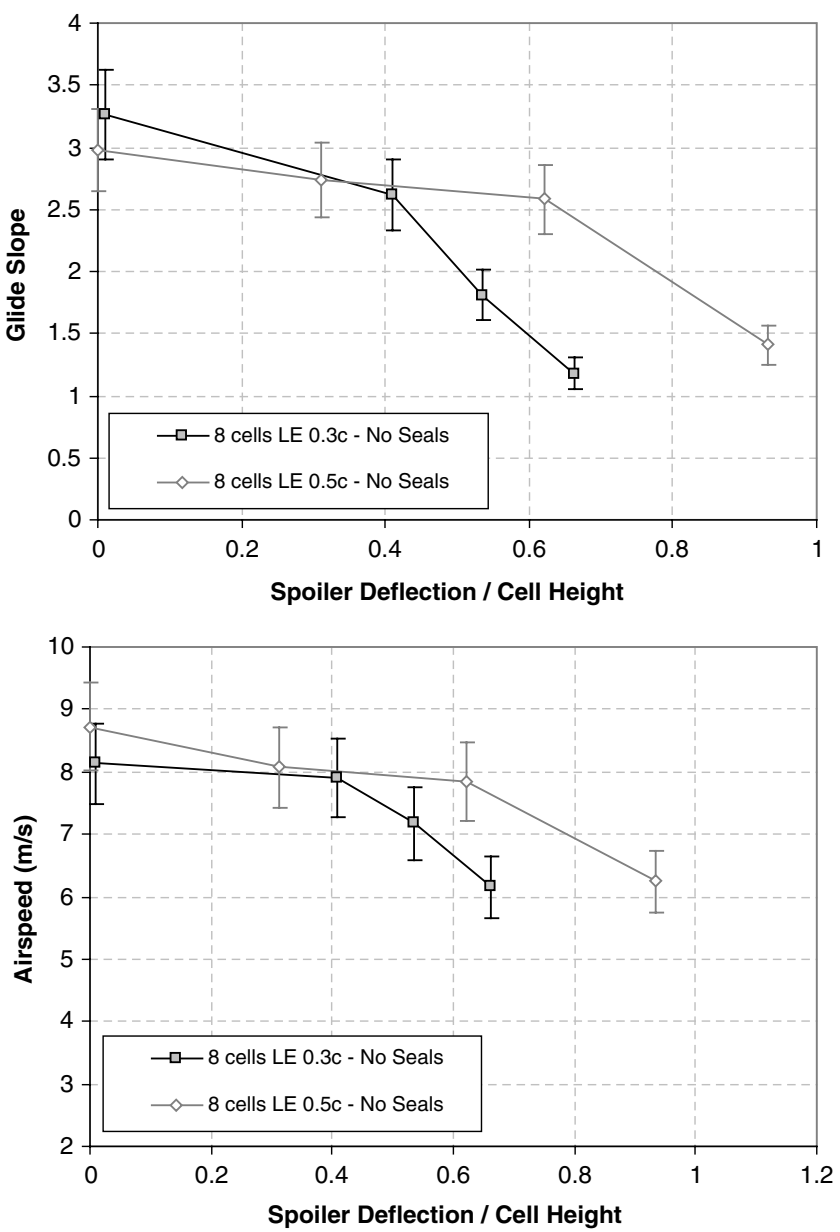

Fig. 13 Effect of moving spoilers aft of nominal location.

slits at $50 \%$ chord, but a much higher spoiler deflection is required. The simple explanation is that the flow over a smaller portion of the wing is affected when the slits are moved aft, so the spoiler becomes less effective.

The slit locations were also moved forward to $15 \%$ of the canopy chord. This configuration was originally flown with simple, unsealed slits, but the small amount of air leakage and canopy deformation caused by the slits was so detrimental to the canopy performance that the system was unable to climb under power. With sealing slits added, the performance of the canopy improved enough to allow the system climb and obtain data. The effectiveness of the spoiler with slits at $15 \%$ of the canopy chord is compared to the spoiler with slits at $30 \%$ chord in Fig. 14. Both of these configurations use sealing flaps, but it is interesting to note that a maximum glide slope of only 3.0 is achieved with the slits at the $15 \%$ chord location, while the full nominal glide ratio of 3.8 was achieved with the sealed slits at the $30 \%$ chord location. This indicates that the canopy is extremely sensitive to any modifications near the LE. Furthermore, under large deflections of the slits at the $15 \%$ chord location, the entire section of the canopy in front of the slit actually collapses, and the spoiler opening becomes the new ram-air inlet of the cell. This situation produces a similar minimum glide ratio of 1.1 to that achieved with the slits at $30 \%$ chord, but the LE collapse results in a severely deformed canopy shape, which makes the system extremely difficult to control. The canopy does not recover from this collapse when the spoiler actuation line is released. This situation was captured in the photograph in Fig. 15.

The overall effectiveness of the upper-surface spoiler in reducing glide slope as a function of chordwise location is summarized in Fig. 16. To make a fair comparison, the effectiveness of the spoiler with the slits at the $50 \%$ chord location was adjusted by assuming that the nominal glide slope of 3.8 would be reached with the addition of sealing flaps. 

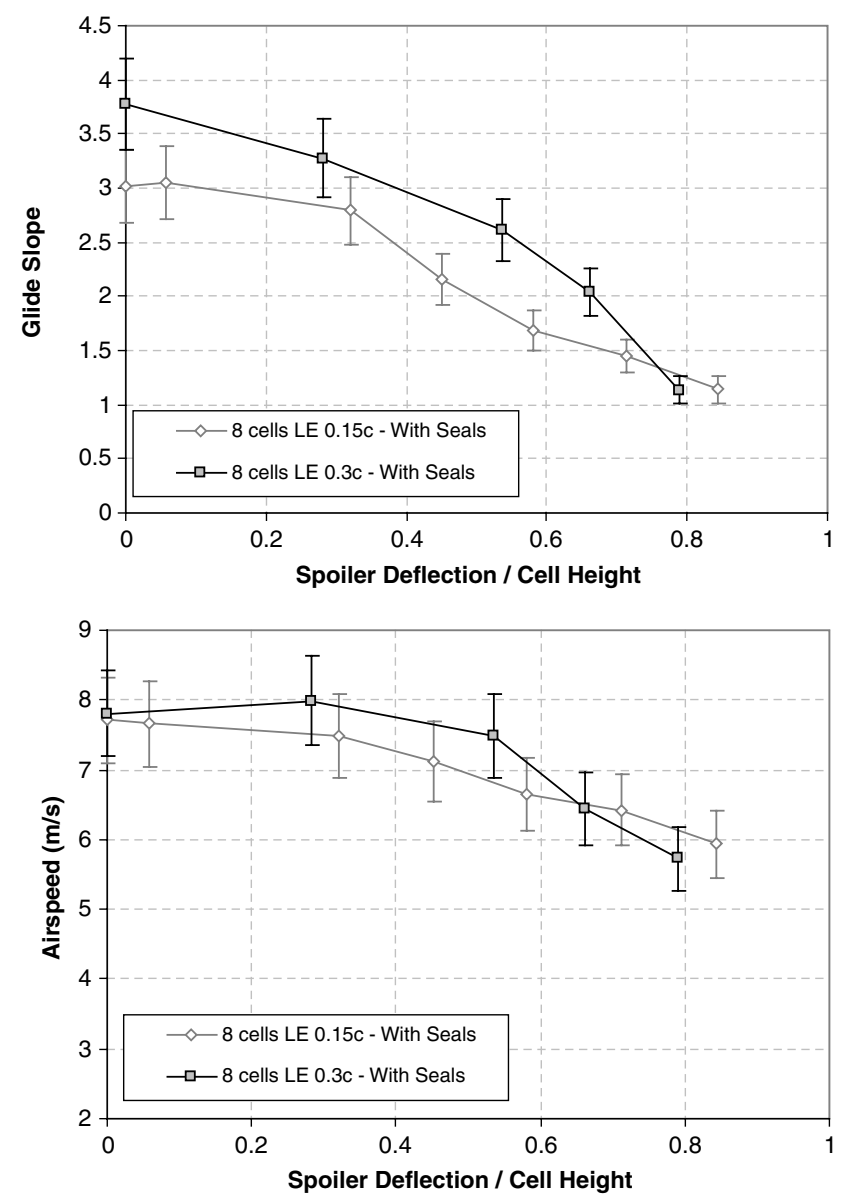

Fig. 14 Effect of moving spoilers forward of nominal location.

\section{B. Asymmetric Spoiler Configurations}

Asymmetric upper-surface spoiler configurations were evaluated for their utility as a lateral control mechanism. All asymmetric spoiler configurations have a simple slit with LE control line attachments and no sealing mechanism. These configurations varied in size from one to four cells on one side of the canopy. Configurations with less than four cells were tested as "inboard" spoilers, where the modified cells begin at the centerline and count outward, and also as "outboard" spoilers, where the modified cells begin with the fourth cell from the centerline and count inward. Figure 17 shows the steady-state turn rate achieved vs. spoiler deflection for all of the tested asymmetric spoiler configurations. Error bars are not shown in this plot because the estimated error for each data point is on the order of $1 \mathrm{deg} / \mathrm{s}$, which is approximately the size of the markers used for

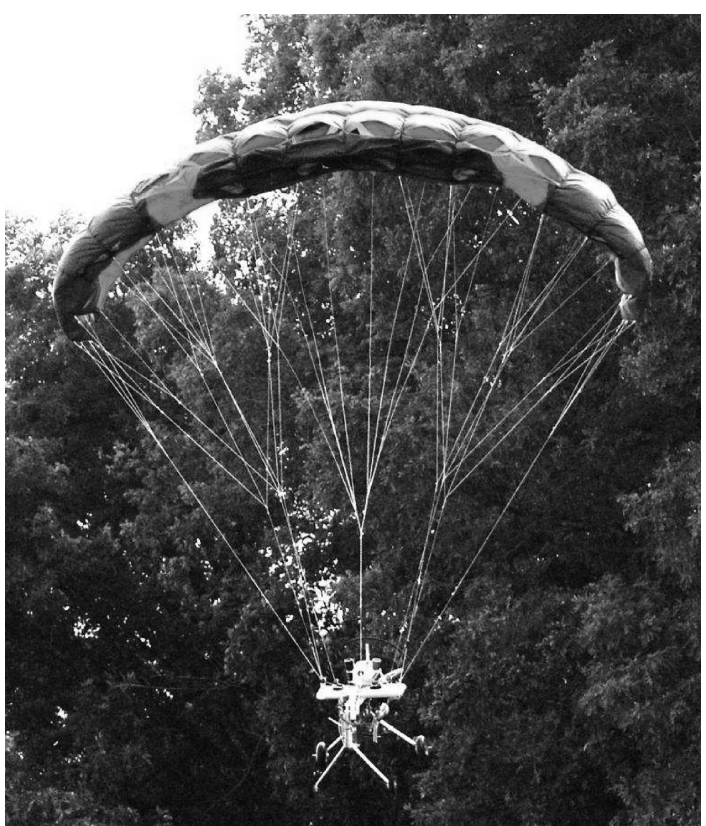

Fig. 15 LE collapse under large forward spoiler deflection.

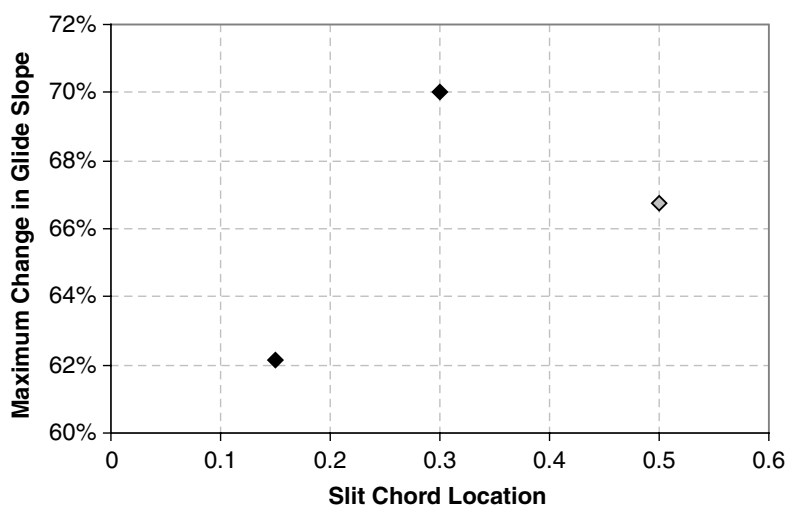

Fig. 16 Glide slope control authority vs. chordwise location.

the data points. Increasing the spoiler size produces a direct increase in turn rate authority and spoilers that are at a greater distance from the centerline ("outboard" vs. "inboard" spoilers) generate higher turn rates at lower slit deflections. The control authority of the majority of the asymmetric spoiler configurations tested is sufficient to achieve a spiral dive. This condition produces a turn rate of $50^{\circ}$ per second for this particular parafoil and payload system, which is considered as the upper limit of the useful turn rate.

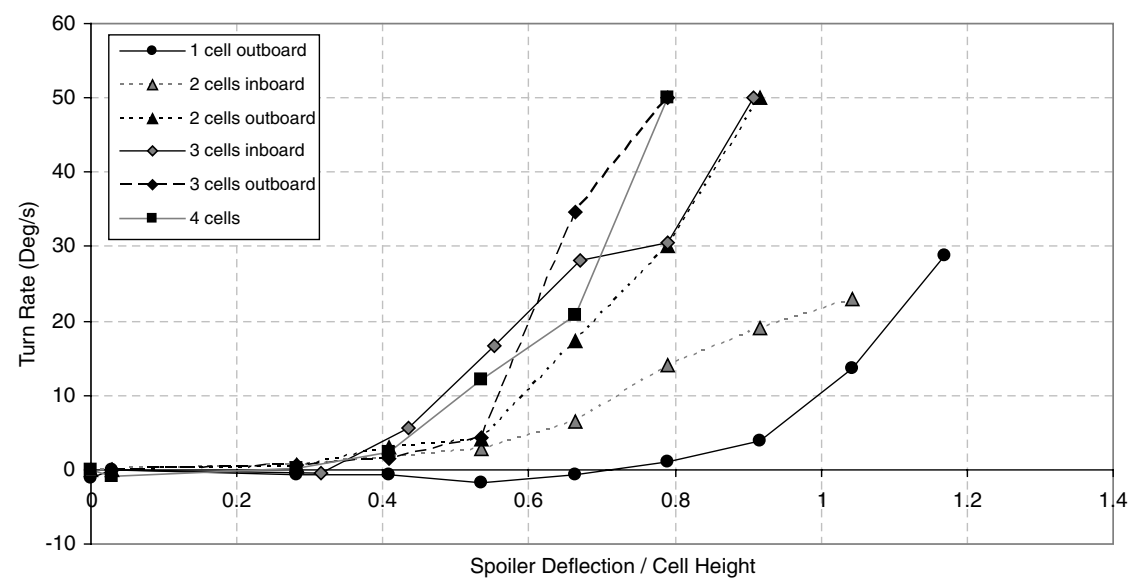

Fig. 17 Turn rate vs. asymmetric spoiler deflection for different configurations. 


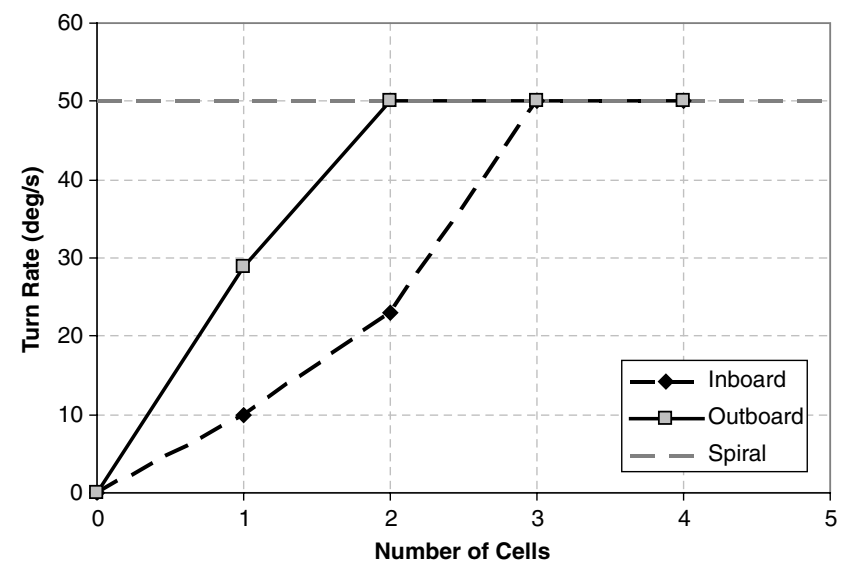

Fig. 18 Lateral control authority vs. asymmetric spoiler configuration.

The maximum turn rate achieved for each asymmetric spoiler configuration is plotted as a function of the number of actuated cells in Fig. 18. The ability to achieve a spiral dive indicates that a particular asymmetric spoiler configuration is able to generate the maximum practical turn rate, which also means that a particular asymmetric spoiler configuration is able to generate the same lateral control authority as the TE brakes. It is interesting to note that for this particular canopy, the actuation of the slits on only two canopy cells is required to achieve the full turn rate capability of this particular parafoil and payload aircraft. Overall, the asymmetric actuation of the upper-surface spoilers is clearly an effective lateral control mechanism.

\section{Conclusions}

Current guided airdrop systems employ right and left brakes that yield effective control of airspeed and turn rate. The addition of control channels, particularly ones that enable effective glide slope control, promises to enable substantial improvements in the impact point accuracy of autonomous airdrop systems. This paper explores the generation of glide slope and turn rate control with the use of a novel control technique for ram-air parafoils based on the idea of upper-surface spoilers. The spoilers vent the canopy's internal ram air through slits in the upper surface to produce an effective aerodynamic spoiler in the flow over the canopy. The spoiler can be actuated symmetrically to produce dramatic reductions in glide slope and asymmetrically for effective lateral control. The spoiler is most effective when the leading edge (LE) side of the slit is deflected and a simple sealing flap is added to prevent air leakage when the spoiler is not actuated. It was shown that actuation of slits across the center $56 \%$ of the canopy was sufficient to achieve the minimum practical glide slope of the tested canopy, and the most effective configuration was able to achieve smooth control of glide slope from 3.8 down to 1.1, a $70 \%$ reduction. The spoiler was found to be most efficient when the slits are located at $30 \%$ of the canopy chord. Moving the slits to $50 \%$ chord diminished the spoiler effectiveness, while moving the slits forward to $15 \%$ chord produced a degradation of canopy performance with no spoiler actuation and an unrecoverable LE collapse under large spoiler actuation. Finally, the asymmetric actuation of the upper-surface canopy spoilers is shown to produce effective lateral control with maximum practical turn rates achieved using as few as two actuated cells. The flight-test results demonstrate that upper-surface spoilers have tremendous potential for improving the control authority of parafoil and payload aircraft. In addition to extremely simple construction and rigging, upper-surface spoilers produce a tremendous effect on the parafoil flight characteristics with much smaller canopy deformations than the standard control mechanism of trailing edge (TE) brake deflection. The resulting reduction in required actuation force implies that the incorporation of upper-surface spoiler control could provide significant cost savings, especially on large parafoil and payload systems.

\section{References}

[1] Ware, G., and Hassell, J., "Wind-Tunnel Investigation of RamAir_Inflated All-Flexible Wings of Aspect Ratios 1.0 to 3.0," NASA TM SX-1923, 1969.

[2] Brown, G., "Parafoil Steady Turn Response to Control Input," AIAA Paper 1993-1241, May 1993.

[3] Petry, G., Behr, R., and Tscharntke, L., "The Parafoil Technology Demonstration (PTD) Project: Lessons Learned and Future Visions," AIAA Paper 1999-1755, June 1999.

[4] Bennett, T., and Fox, R., "Design, Development and Flight Testing of the NASA X-38 7500 Ft2 Parafoil Recovery System," AIAA Paper 2003-2107, May 2003.

[5] Madsen, C., Sostaric, R., and Cerimele, C., "Flight Performance, Aerodynamics, and Simulation Development for the X-38 Parafoil Test Program," AIAA Paper 2003-2108, May 2003.

[6] Slegers, N., and Costello, M., "Aspects of Control for a Parafoil and Payload System," Journal of Guidance, Control, and Dynamics, Vol. 26, No. 6, 2003, pp. 898-905. doi: $10.2514 / 2.6933$

[7] Altmann, H., and Windl, J., "ParaLander: A Medium-Weight Demonstrator for Autonomous, Range-Optimized Aerial Cargo Delivery," AIAA Paper 2005-1627, May 2005.

[8] George, S., Carter, D., Berland, J., Dunker, S., Tavan, S., and Barber, J., "The Dragonfly $4500 \mathrm{~kg}$ Class Guided Airdrop System," AIAA Paper 2005-7095, Sept. 2005.

[9] Jann, T., "Advanced Features for Autonomous Parafoil Guidance, Navigation and Control," AIAA Paper 2005-1642, May 2005.

[10] Slegers, N., Beyer, E., and Costello, M., "Use of Variable Incidence Angle for Glide Slope Control of Autonomous Parafoils," Journal of Guidance, Control, and Dynamics, Vol. 31, No. 3, 2008, pp. 585-596. doi: $10.2514 / 1.32099$

[11] Lippisch, A., "The Development, Design and Construction of Gliders and Sailplanes," NACATM-637, 1931.

[12] Kohlman, D. L., and Brainerd, C. H., "Evaluation of Spoilers for Light Aircraft Flight Path Control," Journal of Aircraft, Vol. 11, No. 8, 1974, pp. 449-456. doi: $10.2514 / 3.60366$

[13] Klein, V., and Morelli, E., Aircraft System Identification: Theory and Practice, AIAA Education Series, AIAA, Reston, VA, 2006, pp. 103 104. 